\title{
Drug resistance markers within an evolving efficacy of anti-malarial drugs in Cameroon: a systematic review and meta-analysis protocol
}

Peter Thelma Ngwa Niba

Universite de Yaounde I

Akindeh M. Nji

Universite de Yaounde I

Marie-Solange Evehe

Universite de Yaounde I

Innocent M. Ali

Universite de Dschang Faculte des Sciences

Palmer Masumbe Netongo

Universite de Yaounde I

Randolph Ngwafor

Universite de Yaounde I

Marcel N. Moyeh

University of Buea

Lesley Ngum Ngum

Universite de Yaounde I

Oliva Ebie Ndum

Universite de Yaounde I

Fon Abongwa Acho

Universite de Yaounde I

Cyrille Mbanwi Mbu'u

Universite de Yaounde I

Dorothy A. Fosah

University de Yaounde I

Barbara Atogho-Tiedeu

Universite de Yaounde I

Olivia Achonduh-Atijegbe

Universite de Yaounde I

Rosine Dadjeu

Universite de Yaounde I

Jean Paul Kengne Chedjou 
Universite de Yaounde I

Jude D. Bigoga

Universite de Yaounde I

Carole Else Eboumbou Moukoko

Universite de Douala Faculte de Medecine et Sciences Pharmaceutiques

\section{Anthony Ajua}

University of Buea

\section{Eric Achidi}

University of Buea

\section{Esther Tallah}

Universite de Yaounde I

Rose G. F. Leke

Universite de Yaounde I

\section{Alexis Tourgordi}

Organisation mondiale de la Sante

\section{Pascal Ringwald}

Organisation mondiale de la Sante

\section{Michael Alifrangis}

University of Copenhagen

Wilfred F. Mbacham ( $\nabla$ wfmbacham@yahoo.com )

Universite de Yaounde I https://orcid.org/0000-0002-3934-3233

\section{Protocol}

Keywords: Malaria, anti-malarial, resistance, mutations, systematic review, meta-analysis, Plasmodium falciparum, Cameroon

Posted Date: September 11th, 2020

DOl: https://doi.org/10.21203/rs.3.rs-26096/v3

License: (c) (1) This work is licensed under a Creative Commons Attribution 4.0 International License. Read Full License 


\section{Abstract}

Background: Cameroon remains a country faced with high malaria burden despite enormous efforts made in the control of the disease. The rapid development and dispersal of mutations associated with anti-malarial drug resistance influenced policy changes from the use of chloroquine, amodiaquine and sulphadoxine-pyrimethamine to the adoption of artemisinin-based combination therapies (ACTs) for the treatment of uncomplicated falciparum malaria. Different studies have identified the frequency of key markers in Plasmodium falciparum associated with drug resistance without a clear picture on the localisation of potential hotspots that may drive the emergence of resistance to the currently used ACTs. This systematic review and meta-analysis aims to determine the prevalence and distribution of $P$. falciparum drug resistance markers within an evolving efficacy of anti-malarial drugs in Cameroon from 1990 to present.

Methods: The PRISMA, PRISMA-P and STREGA statements will be adopted in the quality assessments of studies to be included in this review. The electronic databases of Medline via Pubmed, EMBASE, Google Scholar and Science Direct will be searched by two independent researchers using different MeSH terms and Boolean operators (AND, OR). More so, unpublished data that will be sourced from academic libraries will also be extracted. Quantitative syntheses will be done using the "metaphor" and "meta" commands in the R statistical software package version 3.5.2. Heterogeneity will be assessed using the Cochrane Q and 12 statistics. The random effects model will be used as benchmark in the determination of heterogeneity between studies.

Discussion: The primary outcome of this review is to identify and describe molecular markers conferring drug resistance in Plasmodium falciparum parasites that have been circulating for a period of over 30 years in Cameroon. This review will be able to pool data from previously published and unpublished studies on anti-malarial drug resistance gene mutations. This will provide evidence to support the continuous use of ACTs in the treatment of uncomplicated P. falciparum malaria. Moreover, it is also hoped that potential hotspots driving the emergence and spread of anti-malarial resistance markers will be identified.

Systematic review registration: PROSPERO CRD42020162620

\section{Background}

According to the WHO global statistics, malaria accounted for 228 million cases and 405,000 related deaths in 2018 [1]. Cameroon remains a country with a high malaria burden and impact despite enormous efforts made in the control of the disease [1]. In Cameroon, the rapid emergence and dispersal of drug resistance was responsible for the change of chloroquine (CQ) use as the first-line therapy for treatment of uncomplicated Plasmodium falciparum malaria in 2002 and later amodiaquine (AQ) monotherapy and sulphadoxine-pyrimethamine (SP) between 2002 and 2004 [2]. A major drug policy change was recorded in 2004 when the government of Cameroon officially aligned with the World Health 
Organisation (WHO) recommendations by adopting artesunate-amodiaquine (ASAQ) and later artemether-lumefantrine (AL) in 2006 as first-line treatments for uncomplicated malaria [2,3]. ASAQ and AL drugs are distributed in the proportions of $75 \%$ and $25 \%$ respectively in public health facilities [4]. It important to note that, the AL combination is relatively predominant within the private health facilities and vendors [4]. The efficacy of anti-malarial drugs is linked to the presence or absence of parasites resistant to these drugs in the population. Thus, regular monitoring of drug resistance markers is very essential to malaria control programmes in endemic regions.

The use of advanced molecular biology techniques has greatly facilitated the identification of key amino acid changes in the genes of Plasmodium falciparum chloroquine resistant transporter-PfCrt (C72S, V73K, M74I, N75E, K76T, A220S, Q271E, N326S, I356T, R371I), Plasmodium falciparum multi-drug resistant 1-Pfmdr1 (N86Y, Y184F, S1034C, N1042D, D1246Y, copy number variation), Plasmodium falciparum dihydrofolate reductase-Pfdhfr (A16V, C50R, N51I, C59R, S108N/T) and Plasmodium falciparum dihydropteorate synthase-Pfdhps (I431V, S436A/F, A437G, K540E/N, A581G, A613S/T) associated with resistance to different anti-malarial drugs [5-9]. These include the partner drugs in the much cherished artemisinin based-combination therapies (ACTs). The presence of Pfcrt K76T is associated with increased risk of treatment failure after administration of chloroquine whereas, Pfmdr1 N86Y is associated with both chloroquine and amodiaquine resistance [10]. For sulphadoxinepyrimethamine, the Pfdhfr $(108 \mathrm{~N})$ single mutant, $P f d h f r(511,59 \mathrm{R}, 108 \mathrm{~N})$ triple mutants and PfdhfrPfdhps (51I, 59R, 108N, 437G, 540E) quintuple mutants have been shown to increase the risk of treatment failure [10]. It has also been documented that increased Pfmdr1 copy number is correlated with resistance to mefloquine and reduced sensitivity to lumefantrine [11-13]. A meta-analysis on $A L$ and ASAQ showed opposing effects for Pfcrt K76T and Pfmdr1 N86Y [14]. This was further confirmed by another meta-analysis on the selection of Pfmdr1 NFD haplotype for AL and Pfmdr1 YYY haplotype for ASAQ from samples of efficacy studies conducted in Africa that led to reduced sensitivities of the two drugs [15].

In the period 2009-2010, single nucleotide polymorphisms in the Pfk 13 propeller domain of Cambodian parasite isolates were identified to be associated with delayed parasite clearance of artemisinins [16]. The epicentres driving the emergence and dispersal of artemisinin resistance have been identified in countries within the Greater Mekong sub-region (GMS) namely, Cambodia, China (Yunnan Province), Lao People's Democratic Republic, Myanmar, Thailand and Vietnam [17]. Presently, about 200 nonsynonymous mutations in the $\mathrm{K} 13$ gene have been identified and reported [17-20]. A total of 9 Pfk13 non-synonymous single nucleotide polymorphisms (F446I, N458Y, N458Y, Y493H, R539T, I543T, P553L, R561H, C580Y) have been validated with F446I, R539T, I543T, P574L and C580Y being the most common and with the highest occurrences $[17,19,20]$. There are 11 candidate gene polymorphisms associated with delayed parasite clearance $[17,19,20]$. A number of mutations have also been reported outside the K13 propeller region notably, K189T and E252Q [19,21-23]. In Africa, the highest geographical distribution is A578S $[18,19,24]$ and the presence of R561H mutation has recently been reported in Tanzania [25] and Rwanda [26]. Hence, there are fears that ACT resistance may spread to other regions including subSaharan Africa where malaria is still a major burden, similar to what happened in the past with the 
chloroquine, amodiaquine, and sulphadoxine-pyrimethamine. The rationale for the use of ACT relies on the rapid reduction of the parasite biomass, reduction of transmission (reducing gametocytes), protection of partner drug against resistance, and rapid fever reduction [27].

The effect of drug policy changes on the selection of Plasmodium falciparum anti-malarial drug resistant parasites in Cameroon has not been completely understood. This systematic review and meta-analysis aims to determine the prevalence and distribution of $P$. falciparum drug resistance markers within an evolving efficacy of anti-malarial drugs in Cameroon from January 1990 to present.

\section{Methods/design}

\section{Review questions}

- What is the reported prevalence of falciparum drug resistance markers before and after the adoption of artemisinin-based combination therapies in Cameroon?

- What is the relationship between the ACTs (ASAQ and AL) and prevalence of falciparum drug resistance mutations (chloroquine resistance transporter mutation K76T and multidrug resistance 1 mutation N86Y) in Cameroon?

- What is the impact of the amount of artesunate-amodiaquine (ASAQ), artemether-lumefantrine (AL) and sulphadoxine-pyrimethamine deployed to the different Regions on the evolution of drug resistance markers in Cameroon?

\section{Objectives of the systematic review}

This review aims to:

- Determine the prevalence of falciparum drug resistance markers before and after the adoption of artemisinin-based combination therapies in Cameroon. The key Plasmodium falciparum antimalarial resistance markers shall include: chloroquine resistance transporter ( $P f c r t)$, multi-drug resistance 1 (Pfmdr1), dihydrofolate reductase (Pfdhfr), dihydropteroate synthase (Pfdhps), atpase 6 (Pfatp6), cytochrome b (Pfcytb) and kelch 13 (Pfk13).

- Evaluate the relationship between the efficacy of ACTs (ASAQ and AL) and prevalence of falciparum drug resistance mutations (chloroquine resistance transporter mutation K76T and multidrug resistance 1 mutation N86Y).

- Investigate the impact of the amount of artesunate-amodiaquine (ASAQ), artemether-lumefantrine $(\mathrm{AL})$ and sulphadoxine-pyrimethamine deployed to the different Regions on the evolution of drug resistance markers in Cameroon. 


\section{Registration of the systematic review protocol}

The review protocol has been registered in the International Prospective Register of Systematic Reviews (PROSPERO: http://www.crd.york.ac.uk/prospero). The current review protocol is registered with the number CRD42020162620.

\section{Search strategy and search terms}

An electronic systematic strategy based on the combination of key words will be used to search articles from Medline via Pubmed, EMBASE, Google Scholar, and Science Direct databases. Both interventional and observational studies will be retrieved to be included in the review. The following MeSH search terms will be combined using the Boolean operators "OR" and "AND": "anti-malarial", "drug resistance", "Pfcrt", "Pfmdr1", "Pfmdr1 copy number", "Pfdhfr", "Pfdhps", "Pfatp6", "Pfcytb", "Pfk13", "mutations", "gene polymorphisms", "amino acid changes", "Plasmodium falciparum", "efficacy", "artesunate-amodiaquine", "artemether-lumefantrine”, “Cameroon”.

\section{Additional searches}

The reference lists of published articles will be searched for eligible studies. Authors will be contacted in the case whereby full length articles could not be assessed. Malaria annual reports will be obtained from the Cameroon National Malaria Control Programme (NMCP) and Ministry of Public Health. In addition to published studies, unpublished Medical Doctor (MD), Master of Science (MSc) and Doctor of Philosophy $(\mathrm{PhD})$ theses will be accessed for inclusion in the study.

\section{Eligibility criteria}

\section{Inclusion criteria}

The systematic review and meta-analysis will include the following type of studies:

i) studies published from January 1990 to present; ii) studies on human participants of all ages; iii) original articles of studies that investigated either asymptomatic, uncomplicated or severe Plasmodium falciparum; iv) studies in which Polymerase Chain Reaction (PCR) genotyping of anti-malarial drug molecular resistance markers (Pfcrt, Pfmdr1, Pfdhfr, Pfdhps, Pfcytb, Pfatp6, Pfk13) were done; v) studies written in English or French; vi) studies done within Cameroon; and vii) all multi-centric studies in which Cameroon was one of the sites or cases imported malaria from Cameroon were included in this The selection and inclusion of studies will be done according the population intervention comparator outcome (PICO) format (Additional file 2). 


\section{Exclusion criteria}

The following types of studies will not be included: i) abstracts; ii) studies on in vitro, ex vivo and in vivo anti-malarial drug resistance without Deoxyribonucleic Acid (DNA) sequence genotyping; iii) genetic association studies on Pfcg2 gene; iv) studies on genetic diversity and population structure of Plasmodium falciparum without drug resistance; and v) studies on diagnostic accuracy of methods for detection of $P$. falciparum and mixed Plasmodium species infections.

\section{Review process}

Articles identified from searches of the computerised databases will be screened for eligibility based on title and abstract. Ineligible articles and duplicates will eventually be removed. Full--ength articles of the selected studies will be read to confirm for fulfilling of the inclusion criteria before data extraction began. Two independent reviewers (Peter Thelma Ngwa Niba-PTNN and Lesley Ngum Ngum-LNN) will screen the titles and abstracts to identify potentially eligible studies and data from full-length articles that fulfil the inclusion criteria will be extracted. Discrepancies will be resolved by mutual consent or by independent review from the third researcher (Akindeh Mbuh Nji-AMN). The whole process will be supervised by Wilfred Fon Mbacham (WFM) and Michael Alifrangis (MA).

\section{Data extraction procedure}

Data extraction process will focus on the types of study design (observational versus interventional), year the studies were conducted, study site, sample size, age of participant, genotyping method, genotyping accuracy, drug resistance gene, type of amino acid changes, and prevalence of mutations (Additional file $3)$.

Studies (observational or interventional) published multiple times in similar topics by the same authors will be diligently screened to avoid duplication of data. These studies will be differentiated based on primary variables (anti-malarial drug resistance markers and frequency of single nucleotide polymorphisms) containing the datasets of interest.

Mixed genotypes will be considered as mutants during data collation on frequency of mutations derived from different studies. The Microsoft Excel 2010 (Microsoft Corporation, Redmond, Washington, United States of America) shall be used to design the data extraction sheet. The database in Microsoft Excel will be piloted and validated before completion of the review process. 


\section{Protocol development and selection process}

The Preferred Reporting Items for Systematic Reviews and Meta-analyses (PRISMA) [28,29], Preferred Reporting Items for Systematic Reviews and Meta-analyses Abstracts (PRISMA-Abstracts) [30], Preferred Reporting Items for Systematic Reviews and Meta-analyses Protocol (PRISMA-P) [28,31] and Strengthening the Reporting of Genetic Association Studies (STREGA) [32] statements will be applied in the development of the protocol for this review and meta-analysis. These statements will also be used in the selection of studies on single nucleotide polymorphisms (SNPs) of Plasmodium falciparum antimalarial drug resistance genes (Additional file 1).

\section{Data management}

Data will be managed in the Zotero standalone software version 5.0.56 (Corporation for Digital Scholarship, Vienna, Virginia, United States). Eligible articles will be imported into the software and duplicates removed.

\section{Data analysis, heterogeneity assessment and data interpretation}

Quantitative syntheses will be done using the "metaphor" and "meta" commands in the R statistical software package version 3.5.2 (supported by the RFoundation for Statistical Computing, Vienna, Austria). The heterogeneity of the included studies will be evaluated using the Cochrane $Q$ and $\mathrm{I}^{2}$ statistics. The random effects model will be used as benchmark in the determination of heterogeneity between studies [33]. The $I^{2}$ values will be expressed in percentages. Heterogeneity will be classified as low, moderate and high, with upper limits of $25 \%, 50 \%$ and $75 \%$ for $\mathrm{I}^{2}$, respectively.

Forest plots will be used to present the data on pool prevalence of mutations in anti-malarial drug resistance genes. Sub-group analyses shall be also done to demonstrate the aggregated prevalence of Pfcrt K76T, Pfmdr1 N86Y, Pfdhfr IRN haplotype and Pfk13 gene mutations.

Frequency tables will be used to demonstrate the evolution of key anti-malarial drug resistance markers and quantities of artesunate-amodiaquine, artemether-lumefantrine and sulphadoxine-pyrimethamine deployed to the different regions in Cameroon over the years. The Pearson Chi square test in the International Business Machines Statistical Software Package for Social Sciences (IBM SPSS) version 20.0 software package (IBM Corporation, Armonk, New York, United States of America) will be used to establish the evolution of drug resistance markers over time. Furthermore, the relationship between the efficacy of ACTs (ASAQ and AL) and anti-malarial drug resistance makers (PfCrt 76T and Pfmdr1 86Y) will be assessed on plots. The correlation coefficient ( $r$ ) will be used to assess the relationship between efficacy of ACTs (AL and ASAQ) and prevalence of Pfcrt 76T and Pfmdr1 86Y mutants over time after 
checking for normality using the Shapiro-Wilk test. The level of significance will be set at $p<0.05$ at $95 \%$ confidence interval.

\section{Methodological quality assessment of publication bias}

Publication bias shall be assessed by using the funnel plot and the Egger's regression test. The funnel plot contains the standard error on the $y$-axis and proportion on the $x$-axis.

\section{Discussion}

Malaria can effectively be prevented and cured with the use of appropriate anti-malarial drugs [34]. In the early 2000 , the rapid development and spread of drug resistance markers compelled the Cameroon government to effect malaria treatment changes from monotherapies to artemisinin-based combination therapies in line with the WHO recommendations [2]. Since the ban on the use of chroroquine in many malaria endemic regions, trend analyses have showed the decline of the Pfcrt 76T mutant over the years, thus confirming the re-emergence of chloroquine sensitive parasites [35-37]. Moreover, different studies carried have also demonstrated an opposing effect in the selection of Pfmdr1 YYY triple haplotype for ASAQ and Pfmdr1 NFD triple haplotype for AL $[15,38]$. This can possibly slow down the emergence of drug resistance when the two drugs are used simultaneously [39]. Cameroon may benefit from this phenomenon since ASAQ and AL are used as multiple first-line treatments (MFTs) for uncomplicated Plasmodium falciparum malaria. Furthermore, high prevalence rates of Pfdhfr IRN and Pfdhfr/ Pfdhps IRNG conferring resistance to the anti-folates have been recorded in Equatorial Guinea, a country bordering Cameroon $[40,41]$. In addition, the recent identification of the Pfk 13 R561H gene polymorphisms in Tanzania [25] and Rwanda [26] should be of major concern to malaria endemic countries in sub-Saharan Africa including Cameroon. This is because the artemisinins either used singly or in combination with other drugs is the mainstay in the treatment of malaria globally.

The primary outcome of this review is to identify and describe molecular markers conferring drug resistance in $P$. falciparum parasites that have been circulating for a period of over 3 decades in Cameroon. This review will be able to pool data from previously published and unpublished studies on anti-malarial drug resistance gene mutations. This will provide evidence to support the continuous use of ACTs in the treatment of uncomplicated $P$. falciparum malaria. Furthermore, the data generated from this review will provide baseline information on the design and adoption of a robust anti-malarial drug resistance surveillance system nationwide which is inexistent. A robust surveillance system will be anchored on that the identification of potential hotspots driving the emergence and spread of antimalarial resistance markers.

Logically, there is a possibility to find variance across studies duration the collation and analysis processes. The origin of heterogeneity between studies can either be clinical, methodological or statistical. The Cochrane $\mathrm{Q}$ and $\mathrm{I}^{2}$ will be adopted to determine whether there are genuine differences 
underlying the findings of the studies, or whether the variation in results is compatible with chance alone. The degree of heterogeneity in this study may have an impact on the accuracy, interpretation and acceptability of findings.

\section{List Of Abbreviations}

ACT: Artemisinin-based combination therapy, AL: Artemether-lumefantrine, ASAQ: Artesunateamodiaquine, DNA: Deoxyribonucleic Acid, MD: Medical Doctor, NMCP: National Malaria Control Programme, MSc: Master of Science, PCR: Polymerase Chain Reaction, PhD: Doctor of Philosophy, Pfcrt. Plasmodium falciparum chloroquine resistance transporter, Pfmdr1: Plasmodium falciparum multi-drug resistance 1, Pfdhfr. Plasmodium falciparum dihydrofolate reductase, Pfdhps: Plasmodium falciparum dihydropteroate synthase, Pfcytb: Plasmodium falciparum cytochrome b, Pfatp6: Plasmodium falciparum atpase 6, Pfk13: Plasmodium falciparum kelch 13, PRISMA: Preferred Reporting Items for Systematic Reviews and Meta-analyses, PRISMA-Abstracts: Preferred Reporting Items for Systematic Reviews and Meta-analyses Abstracts, PRISMA-P: Preferred Reporting Items for Systematic Reviews and Meta-analyses protocol, r: Correlation coefficient, SP: Sulphadoxine-pyrimethamine, SPAQ: Sulphadoxinepyrimethamine-amodiaquine, STREGA: Strengthening the Reporting of Genetic Association Studies, WHO: World Health Organisation

\section{Declarations}

\section{Ethics approval and consent to participate}

Not applicable since it is a systematic review and meta-analysis.

\section{Consent for publication}

Not applicable.

\section{Availability of data and materials}

Not applicable as this is still a systematic review and meta-analysis protocol with no data available for publication.

\section{Competing interests}

The authors declare that they have no competing interests. 


\section{Funding}

WFM, AMN, IMA and PTNN are supported by the Malaria Research Capacity Development in West and Central Africa (MARCAD) Consortium through the Developing Excellence in Leadership, Training and Science (DELTAS) Africa Initiative [grant \# DEL-15-010] to the University of Yaounde I. The DELTAS Africa Initiative is an independent funding scheme of the African Academy of Sciences (AAS)'s Alliance for Accelerating Excellence in Science in Africa (AESA) and supported by the New Partnership for Africa's Development Planning and Coordinating Agency (NEPAD Agency) with funding from the Wellcome Trust [grant \# 107741/A/15/Z] and the United Kingdom (UK) government.

\section{Authors' contributions}

WFM conceived the research and coordinated the study. AMN and PTNN drafted the manuscript, critically reviewed the manuscript, and wrote the final manuscript. The authors WFM, MA, MSE, IMA, PMN, RN, MNM, LNN, OEN, FAA, CMM, DAF, BAT, OAA, RD, JPC, JDB, CEEM, AA, EA, ET, RGFL, AT and PR proof read the manuscript. All authors read and approved the final manuscript.

\section{Acknowledgements}

Not applicable.

\section{Authors' information}

${ }^{1}$ MARCAD-DELTAS Programme, Laboratory for Public Health Research Biotechnologies, University of Yaounde I, Yaounde, Cameroon. ${ }^{2}$ The Biotechnology Centre, University of Yaounde I, Yaounde, Cameroon. ${ }^{3}$ Department of Biochemistry, Faculty of Science, University of Yaounde I, Yaounde, Cameroon. ${ }^{4}$ Department of Biochemistry, Faculty of Medicine and Biomedical Sciences, University of Yaounde I, Yaounde, Cameroon. Institute of Medical Research and Medicinal Plant Studies, Ministry of Scientific Research and Innovation, Yaounde, Cameroon. ${ }^{6}$ Department of Biochemistry, Faculty of Science, University of Dschang, Dschang, Cameroon. ${ }^{7}$ National Malaria Control Programme, Ministry of Public Health, Yaounde, Cameroon. ${ }^{8}$ Université des Montagnes, Banganté, West Region, Cameroon. ${ }^{9}$ Department of Biochemistry and Molecular Biology, Faculty of Science, University of Buea, Buea, Cameroon.

${ }^{10}$ Department of Microbiology, University of Yaounde I, Yaounde, Cameroon. ${ }^{11}$ Faculty of Medicine and Pharmaceutical Sciences, University of Douala, Cameroon. ${ }^{12}$ Malaria Research Service, Centre Pasteur Cameroon, Yaounde, Cameroon. ${ }^{13}$ Malaria Consortium-Cameroon Coalition Against Malaria, Yaounde, 
Cameroon. ${ }^{14}$ The Cameroon Office of the World Health Organisation, Yaounde, Cameroon. ${ }^{15} \mathrm{Global}$ Malaria Programme, World Health Organisation, Geneva, Switzerland. ${ }^{16}$ Centre for Medical Parasitology, Department of Immunology and Microbiology, Faculty of Health and Medical Sciences, University of Copenhagen. ${ }^{17}$ Department of Infectious Diseases, Copenhagen University Hospital, Denmark.

\section{Disclaimer}

The views expressed in this publication are those of the author (s) and not necessarily those of AAS, NEPAD Agency, Wellcome Trust or the UK government or the WHO Geneva.

\section{References}

1. World Health Organisation (WHO): World Malaria Report 2019. https://www.who.int/publicationsdetail/world-malaria-report-2019. Accessed on $8^{\text {th }}$ December, 2019.

2. Cameroon National Malaria Control Programme (NMCP) annual report of activities 2006.

3. World Health Organisation (WHO): World Malaria Report 2008. http://www.who.int/malaria/publications/atoz/9789241563697/en/. Accessed on $10^{\text {th }}$ August, 2017.

4. Sayang C, Gausseres M, Vernazza-Licht N, Malvy D, Bley D, Millet P. Treatment of malaria from monotherapy to artemisinin-based combination therapy by health professionals in urban health facilities in Yaoundé, central province, Cameroon. Malar J [Internet]. 2009 Jul 29;8:176-176. Available from: https://www.ncbi.nlm.nih.gov/pubmed/19640292.

5. Djimdé A, Doumbo OK, Cortese JF, Kayentao K, Doumbo S, Diourté Y, et al. A Molecular Marker for Chloroquine-Resistant Falciparum Malaria. N Engl J Med [Internet]. 2001 Jan 25 [cited 2019 May 21];344(4):257-63. Available from: http://www.nejm.org/doi/abs/10.1056/NEJM200101253440403.

6. Cowman AF, Morry MJ, Biggs BA, Cross GA, Foote SJ. Amino acid changes linked to pyrimethamine resistance in the dihydrofolate reductase-thymidylate synthase gene of Plasmodium falciparum. Proc Natl Acad Sci U S A [Internet]. 1988 Dec;85(23):9109-13. Available from: https://www.ncbi.nlm.nih.gov/pubmed/3057499.

7. Foote SJ, Galatis D, Cowman AF. Amino acids in the dihydrofolate reductase-thymidylate synthase gene of Plasmodium falciparum involved in cycloguanil resistance differ from those involved in pyrimethamine resistance. Proc Natl Acad Sci [Internet]. 1990 Apr 1 [cited 2019 Dec 5];87(8):3014-7. Available from: http://www.pnas.org/cgi/doi/10.1073/pnas.87.8.3014.

8. Peterson DS, Walliker D, Wellems TE. Evidence that a point mutation in dihydrofolate reductasethymidylate synthase confers resistance to pyrimethamine in falciparum malaria. Proc Natl Acad Sci [Internet]. 1988 Dec 1;85(23):9114. Available from: http://www.pnas.org/content/85/23/9114.abstract. 
9. Triglia T, Menting JGT, Wilson C, Cowman AF. Mutations in dihydropteroate synthase are responsible for sulfone and sulfonamide resistance in Plasmodium falciparum. Proc Natl Acad Sci [Internet]. 1997 Dec 9 [cited 2019 Dec 5];94(25):13944-9. Available from: http://www.pnas.org/cgi/doi/10.1073/pnas.94.25.13944.

10. Picot S, Olliaro P, de Monbrison F, Bienvenu A-L, Price RN, Ringwald P. A systematic review and metaanalysis of evidence for correlation between molecular markers of parasite resistance and treatment outcome in falciparum malaria. Malar J [Internet]. 2009 May 4;8:89-89. Available from: https://www.ncbi.nlm.nih.gov/pubmed/19413906.

11. Lim P, Alker AP, Khim N, Shah NK, Incardona S, Doung S, et al. Pfmdr1 copy number and arteminisin derivatives combination therapy failure in falciparum malaria in Cambodia. Malar J [Internet]. 2009 Jan 12;8:11-11. Available from: https://pubmed.ncbi.nlm.nih.gov/19138391.

12. Sidhu ABS, Uhlemann A-C, Valderramos SG, Valderramos J-C, Krishna S, Fidock DA. Decreasing pfmdr1 copy number in Plasmodium falciparum malaria heightens susceptibility to mefloquine, lumefantrine, halofantrine, quinine, and artemisinin. J Infect Dis [Internet]. 2006/07/11 ed. 2006 Aug 15;194(4):528-35. Available from: https://pubmed.ncbi.nlm.nih.gov/16845638.

13. Simpson JA, Jamsen KM, Anderson TJC, Zaloumis S, Nair S, Woodrow C, et al. Nonlinear mixedeffects modelling of in vitro drug susceptibility and molecular correlates of multidrug resistant Plasmodium falciparum. PloS One [Internet]. 2013 Jul 24;8(7):e69505-e69505. Available from: https://pubmed.ncbi.nlm.nih.gov/23894496.

14. Venkatesan M, Gadalla NB, Stepniewska K, Dahal P, Nsanzabana C, Moriera C, et al. Polymorphisms in Plasmodium falciparum chloroquine resistance transporter and multidrug resistance 1 genes: parasite risk factors that affect treatment outcomes for $P$. falciparum malaria after artemetherlumefantrine and artesunate-amodiaquine. Am J Trop Med Hyg [Internet]. 2014/07/21 ed. 2014 Oct;91(4):833-43. Available from: https://pubmed.ncbi.nlm.nih.gov/25048375.

15. Okell LC, Reiter LM, Ebbe LS, Baraka V, Bisanzio D, Watson OJ, et al. Emerging implications of policies on malaria treatment: genetic changes in the Pfmdr-1 gene affecting susceptibility to artemether-lumefantrine and artesunate-amodiaquine in Africa. BMJ Glob Health [Internet]. 2018 Oct 19;3(5):e000999-e000999. Available from: https://www.ncbi.nlm.nih.gov/pubmed/30397515.

16. Ariey F, Witkowski B, Amaratunga C, Beghain J, Langlois A-C, Khim N, et al. A molecular marker of artemisinin-resistant Plasmodium falciparum malaria. Nature [Internet]. 2014 Jan 2;505(7481):50-5. Available from: https://www.ncbi.nlm.nih.gov/pubmed/24352242.

17. Ménard D, Khim N, Beghain J, Adegnika AA, Shafiul-Alam M, Amodu O, et al. A Worldwide Map of Plasmodium falciparum K13-Propeller Polymorphisms. N Engl J Med [Internet]. 2016 Jun 23;374(25):2453-64. Available from: https://www.ncbi.nlm.nih.gov/pubmed/27332904.

18. Kamau E, Campino S, Amenga-Etego L, Drury E, Ishengoma D, Johnson K, et al. K13-Propeller Polymorphisms in Plasmodium falciparum Parasites From Sub-Saharan Africa. J Infect Dis [Internet]. 2014 Nov 2 [cited 2019 Jun 5]; Available from: https://academic.oup.com/jid/articlelookup/doi/10.1093/infdis/jiu608. 
19. Ocan M, Akena D, Nsobya S, Kamya MR, Senono R, Kinengyere AA, et al. K13-propeller gene polymorphisms in Plasmodium falciparum parasite population in malaria affected countries: a systematic review of prevalence and risk factors. Malar J [Internet]. 2019 Mar 7;18(1):60. Available from: https://doi.org/10.1186/s12936-019-2701-6.

20. World Health Organization. Artemisinin resistance and artemisinin-based combination therapy efficacy: status report [Internet]. Geneva: World Health Organization; 2018. Available from: https://apps.who.int/iris/handle/10665/274362.

21. Safeukui I, Fru-Cho J, Mbengue A, Suresh N, Njimoh DL, Bumah VV, et al. Characterization of polymorphisms in <em>Plasmodium falciparum</em> artemisinin resistance marker $<$ em>kelch13</em> in asymptomatic infections in a rural area of Cameroon. bioRxiv [Internet]. 2017 Jan 1;148999. Available from: http://biorxiv.org/content/early/2017/06/12/148999.abstract.

22. Torrentino-Madamet M, Fall B, Benoit N, Camara C, Amalvict R, Fall M, et al. Limited polymorphisms in k13 gene in Plasmodium falciparum isolates from Dakar, Senegal in 2012-2013. Malar J [Internet]. 2014 Dec 4;13:472-472. Available from: https://www.ncbi.nlm.nih.gov/pubmed/25471113.

23. Apinjoh TO, Mugri RN, Miotto O, Chi HF, Tata RB, Anchang-Kimbi JK, et al. Molecular markers for artemisinin and partner drug resistance in natural Plasmodium falciparum populations following increased insecticide treated net coverage along the slope of mount Cameroon: cross-sectional study. Infect Dis Poverty [Internet]. 2017 Dec [cited 2019 Jun 5];6(1). Available from: http://idpjournal.biomedcentral.com/articles/10.1186/s40249-017-0350-y.

24. Feng J, Kong X, Xu D, Yan H, Zhou H, Tu H, et al. Investigation and Evaluation of Genetic Diversity of Plasmodium falciparum Kelch 13 Polymorphisms Imported From Southeast Asia and Africa in Southern China. Front Public Health [Internet]. 2019 Apr 24;7:95-95. Available from: https://www.ncbi.nlm.nih.gov/pubmed/31069209.

25. Bwire GM, Ngasala B, Mikomangwa WP, Kilonzi M, Kamuhabwa AAR. Detection of mutations associated with artemisinin resistance at k13-propeller gene and a near complete return of chloroquine susceptible falciparum malaria in Southeast of Tanzania. Sci Rep [Internet]. 2020 Feb 26;10(1):3500. Available from: https://doi.org/10.1038/s41598-020-60549-7.

26. Uwimana A, Legrand E, Stokes BH, Ndikumana J-LM, Warsame M, Umulisa N, et al. Emergence and clonal expansion of in vitro artemisinin-resistant Plasmodium falciparum kelch13 R561H mutant parasites in Rwanda. Nat Med [Internet]. 2020 Aug 3 [cited 2020 Aug 12]; Available from: http://www.nature.com/articles/s41591-020-1005-2.

27. Okell LC, Drakeley CJ, Bousema T, Whitty CJM, Ghani AC. Modelling the Impact of Artemisinin Combination Therapy and Long-Acting Treatments on Malaria Transmission Intensity. PLOS Med [Internet]. 2008 Nov 25;5(11):e226. Available from: https://doi.org/10.1371/journal.pmed.0050226.

28. Moher D, Shamseer L, Clarke M, Ghersi D, Liberati A, Petticrew M, et al. Preferred reporting items for systematic review and meta-analysis protocols (PRISMA-P) 2015 statement. Syst Rev [Internet]. 2015 Jan 1;4(1):1-1. Available from: https://www.ncbi.nlm.nih.gov/pubmed/25554246. 
29. Liberati A, Altman DG, Tetzlaff J, Mulrow C, Gotzsche PC, loannidis JPA, et al. The PRISMA statement for reporting systematic reviews and meta-analyses of studies that evaluate healthcare interventions: explanation and elaboration. BMJ [Internet]. 2009 Dec 4 [cited 2019 Dec 10];339(jul21 1):b2700b2700. Available from: http://www.bmj.com/cgi/doi/10.1136/bmj.b2700.

30. Beller EM, Glasziou PP, Altman DG, Hopewell S, Bastian H, Chalmers I, et al. PRISMA for Abstracts: Reporting Systematic Reviews in Journal and Conference Abstracts. PLoS Med [Internet]. 2013 Apr 9 [cited 2019 Dec 15];10(4):e1001419. Available from: https://dx.plos.org/10.1371/journal.pmed.1001419.

31. Shamseer L, Moher D, Clarke M, Ghersi D, Liberati A, Petticrew M, et al. Preferred reporting items for systematic review and meta-analysis protocols (PRISMA-P) 2015: elaboration and explanation. BMJ [Internet]. 2015 Jan 2 [cited 2019 Dec 10];349(jan02 1):g7647-g7647. Available from: http://www.bmj.com/cgi/doi/10.1136/bmj.g7647.

32. Little J, Higgins JPT, loannidis JPA, Moher D, Gagnon F, von Elm E, et al. STrengthening the REporting of Genetic Association Studies (STREGA) - An Extension of the STROBE Statement. PLOS Med [Internet]. 2009 Feb 3;6(2):e1000022. Available from: https://doi.org/10.1371/journal.pmed.1000022.

33. Ryan R; Cochrane Consumers and Communication Review Group. Heterogeneity and subgroup analyses in Cochrane Consumers and Communication Group reviews: planning the analysis at protocol stage. http://cccrg.cochrane.org,

December 2016. Accessed on $12^{\text {th }}$ February, 2019.

34. World Health Organisation (WHO). Guidelines for the treatment of malaria. Third edition April 2015. https://www.who.int/malaria/publications/atoz/9789241549127/en/. Accessed on 15th July, 2020.

35. Duah NO, Matrevi SA, de Souza DK, Binnah DD, Tamakloe MM, Opoku VS, et al. Increased pfmdr1 gene copy number and the decline in pfcrt and pfmdr1 resistance alleles in Ghanaian Plasmodium falciparum isolates after the change of anti-malarial drug treatment policy. Malar J [Internet]. 2013 Oct 30;12:377-377. Available from: https://www.ncbi.nlm.nih.gov/pubmed/24172030.

36. Dagnogo O, Ako AB, Ouattara L, Dago ND, Coulibaly DN, Touré AO, et al. Towards a re-emergence of chloroquine sensitivity in Côte d'Ivoire? Malar J [Internet]. 2018 Nov 7;17(1):413. Available from: https://doi.org/10.1186/s12936-018-2551-7.

37. Wamae K, Okanda D, Ndwiga L, Osoti V, Kimenyi KM, Abdi Al, et al. No Evidence of Plasmodium falciparum k13 Artemisinin Resistance-Conferring Mutations over a 24-Year Analysis in Coastal Kenya but a Near Complete Reversion to Chloroquine-Sensitive Parasites. Antimicrob Agents Chemother [Internet]. 2019 Oct 7 [cited 2019 Dec 10];63(12):e01067-19, /aac/63/12/AAC.0106719.atom. Available from: http://aac.asm.org/lookup/doi/10.1128/AAC.01067-19.

38. Sondo P, Derra K, Diallo Nakanabo S, Tarnagda Z, Kazienga A, Zampa O, et al. ArtesunateAmodiaquine and Artemether-Lumefantrine Therapies and Selection of Pfcrt and Pfmdr1 Alleles in Nanoro, Burkina Faso. PloS One [Internet]. 2016 Mar 31;11(3):e0151565-e0151565. Available from: https://www.ncbi.nlm.nih.gov/pubmed/27031231. 
39. Boni MF, Smith DL, Laxminarayan R. Benefits of using multiple first-line therapies against malaria. Proc Natl Acad Sci [Internet]. 2008 Sep 16;105(37):14216. Available from: http://www.pnas.org/content/105/37/14216.abstract.

40. Berzosa P, Esteban-Cantos A, García L, González V, Navarro M, Fernández T, et al. Profile of molecular mutations in pfdhfr, pfdhps, pfmdr1, and pfcrt genes of Plasmodium falciparum related to resistance to different anti-malarial drugs in the Bata District (Equatorial Guinea). Malar J [Internet]. 2017 Jan 13;16(1):28. Available from: https://doi.org/10.1186/s12936-016-1672-0.

41. Jiang T, Chen J, Fu H, Wu K, Yao Y, Eyi JUM, et al. High prevalence of Pfdhfr-Pfdhps quadruple mutations associated with sulfadoxine-pyrimethamine resistance in Plasmodium falciparum isolates from Bioko Island, Equatorial Guinea. Malar J [Internet]. 2019 Mar 26;18(1):101. Available from: https://doi.org/10.1186/s12936-019-2734-x.

\section{Supplementary Files}

This is a list of supplementary files associated with this preprint. Click to download.

- Additionalfile1PRISMAP2015Checklist.docx

- Additionalfile1PRISMAP2015Checklist.docx

- Additionalfile2Pilotedscreeningchecklist.docx

- Additionalfile2Pilotedscreeningchecklist.docx

- Additionalfile3Piloteddataextractionform.xlsx

- Additionalfile3Piloteddataextractionform.xlsx 